\title{
Orthogonal and projected orthogonal matched filter detection ${ }^{\text {is }}$
}

\author{
Yonina C. Eldar ${ }^{\mathrm{a}, *, 1}$, Alan V. Oppenheim ${ }^{\mathrm{b}}$, Dianne Egnor ${ }^{\mathrm{c}}$

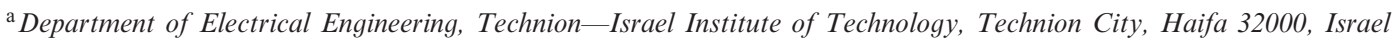 \\ ${ }^{\mathrm{b}}$ Research Laboratory of Electronics, Massachusetts Institute of Technology, Room 36-615, Cambridge, MA 02139, USA \\ ${ }^{\mathrm{c}}$ BAE Systems, P.O. Box 868, MER15-2350, Nashua, NH 03061-0868, USA
}

Received 27 June 2002; received in revised form 25 November 2003

\begin{abstract}
This paper considers the generic problem of detecting in the presence of additive noise, which one from a set of known signals has been received. In place of the classical matched filter (MF) receiver we propose a modified receiver. When the transmitted signals are linearly independent this receiver is referred to as an orthogonal matched filter (OMF) receiver, and when the transmitted signals are linearly dependent it is referred to as a projected orthogonal matched filter (POMF) receiver. Two equivalent representations of the receiver are developed with different implications in terms of implementation. In the first, the demodulator consists of a MF demodulator followed by an optimal whitening transformation on a space formed by the transmitted signals, that optimally decorrelates the MF outputs prior to detection. In the second, the demodulator consists of a bank of correlators with correlating signals that are projections of a set of orthogonal signals, and are closest in a least-squares sense to the transmitted signals. We provide simulation results that suggest that in certain cases the OMF and POMF receivers can significantly increase the probability of correct detection over the MF receiver in non-Gaussian noise with only a minor impact on performance in Gaussian noise.
\end{abstract}

(C) 2003 Elsevier B.V. All rights reserved.

Keywords: Matched-filter detection; Non-Gaussian noise; Multi-signature detection; Least-squares orthogonalization; MMSE whitening

\section{Introduction}

A generic problem which has been studied extensively is that of detecting which one of a set of known

\footnotetext{
This research was supported in part through collaborative participation in the Advanced Sensors Consortium sponsored by the US Army Research Laboratory under Cooperative Agreement DAAL01-96-2-0001.

* Corresponding author. Tel.: +972-4-829-3256; fax: +972-4829-5757.

E-mail addresses: yonina@ee.technion.ac.il (Y.C. Eldar), avo@mit.edu (A.V. Oppenheim), dianne.e.egnor@baesystems. com (D. Egnor).

${ }^{1}$ The author was with the Research Laboratory of Electronics, Massachusetts Institute of Technology, Cambridge, MA, USA.
}

equal-energy signals is received over an additive noise channel. This problem arises in a wide variety of contexts including target classification, signature analysis, and other multi-signature problems.

When the additive noise is white and Gaussian and the signals have equal prior probabilities, it is well known (see, e.g., [26,27]) that the receiver which maximizes the probability of correct detection consists of a demodulator comprised of a bank of correlators with correlating signals equal to the transmitted set, followed by a detector which chooses as the detected signal the one for which the output of the correlator is maximum. This demodulator, referred to as a matched filter (MF) demodulator, is alternatively implemented 
using a bank of filters with impulse responses matched to the transmitted set.

If the noise is not Gaussian, then the MF receiver does not necessarily maximize the probability of correct detection. However, it is still used as the receiver of choice in many applications since the optimal detector for non-Gaussian noise is typically nonlinear (see, e.g., [23] and references therein), and depends on the noise distribution which may not be known. One justification often given for its use is that if a signal is corrupted by Gaussian or non-Gaussian additive white noise, then the filter matched to that signal maximizes the output signal-to-noise ratio (SNR) from all linear filters [26].

In this paper we propose a modification of the MF receiver which again consists of a bank of correlators followed by the same detector used in the MF receiver. This receiver depends only on the transmitted signals, so that it does not require knowledge of the noise distribution or the channel SNR. The simulations presented in Section 7 strongly suggest that when the additive noise is non-Gaussian this modified receiver can significantly increase the probability of correct detection over the MF receiver, particularly when the probability of correct detection with the MF is marginal. When the additive noise is Gaussian, the reduction in performance over the MF receiver is minor. Applications of these ideas to a communication problem have also been explored [8,14].

Two equivalent representations of the modified demodulator are developed in the paper. In the first, developed in Sections 3 and 4, the demodulator consists of a MF demodulator followed by an optimal whitening transformation on a space formed by the transmitted signals [15]. This whitening transformation is designed to optimally decorrelate the outputs of the MF prior to detection. In the second, developed in Section 5, the demodulator consists of a bank of correlators with correlating signals that are projections of a set of orthogonal signals, and are closest in a least-squares sense to the transmitted signals. These two representations are mathematically equivalent but may have different implications in terms of implementation.

The development of the modified receiver considers separately the case in which the transmitted signals are linearly independent and the case in which they are linearly dependent. The receiver for the first case is referred to as an orthogonal matched filter (OMF) receiver and the receiver for the second case as a projected orthogonal matched filter (POMF) receiver. This choice of terminology is based on the interpretation of the receivers in terms of orthogonal signals, developed in Section 5.

In Section 6 we show that if the transmitted signals have a strong symmetry property called geometric uniformity $[6,9,17]$, then the OMF and POMF demodulators maximize the total output SNR subject to the constraint that the outputs of the demodulator are uncorrelated on the appropriate space. This provides some additional justification for this class of receivers.

Before proceeding to the detailed development, in Section 2 we first provide an overview of the notation, problem and main results.

\section{Problem formulation and main results}

We denote vectors in $\mathscr{R}^{m}$ ( $m$ arbitrary) by boldface lowercase letters, and matrices in $\mathscr{R}^{m \times m}$ by boldface uppercase letters. General linear transformations are denoted by uppercase letters. $P_{\mathscr{U}}$ denotes the orthogonal projection operator onto the subspace $\mathscr{U}$, and $\mathbf{I}_{m}$ denotes the $m \times m$ identity matrix. The adjoint and the Moore-Penrose pseudo-inverse of a transformation are denoted by $(\cdot)^{*}$ and $(\cdot)^{\dagger}$ respectively, and $(\widehat{\cdot})$ denotes an optimal signal or transformation. The unique symmetric square root of a matrix is denoted by $(\cdot)^{-1 / 2}$. The variance of a random variable $a$ is denoted by $\operatorname{var}(a)$, and the cross-covariance of $a$ and $b$ is denoted by $\operatorname{cov}(a, b)$. A prime attached to a random variable or vector denotes the variable or vector with the mean subtracted, e.g., $\mathbf{a}^{\prime}=\mathbf{a}-E(\mathbf{a})$. The set transformation $X: \mathscr{R}^{m} \rightarrow \mathscr{H}$ corresponding to a set of signals $\left\{x_{k}(t) \in \mathscr{H}, 1 \leqslant k \leqslant m\right\}$ is defined as ${ }^{2}$

$X \mathbf{z}=\sum_{k=1}^{m} x_{k}(t) z_{k}$

for any vector $\mathbf{z} \in \mathscr{R}^{m}$, where $z_{k}$ denotes the $k$ th element of z. The adjoint transformation $X^{*}: \mathscr{H} \rightarrow$ $\mathscr{R}^{m}$ is defined by $\langle X \mathbf{z}, y(t)\rangle=\left\langle\mathbf{z}, X^{*} y(t)\right\rangle$ for any $\mathbf{z} \in \mathscr{R}^{m}, y(t) \in \mathscr{H}$, which implies that if $\mathbf{a}=X^{*} y(t)$

\footnotetext{
${ }^{2}$ In [2] the set transformation corresponding to the signals $x_{k}(t)$ is referred to as a hypervector and denoted by $\left(x_{1}(t), \ldots, x_{m}(t)\right)$. We prefer the more compact transformation notation.
} 


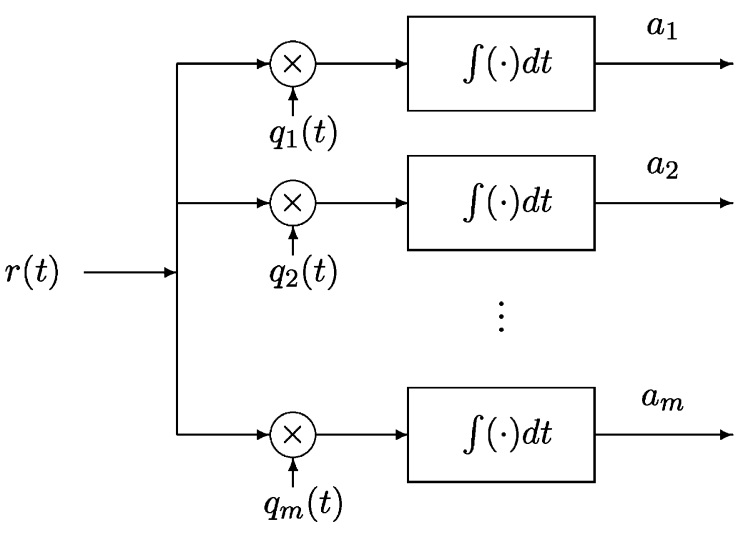

Fig. 1. Correlation demodulator.

then

$a_{k}=\left\langle x_{k}(t), y(t)\right\rangle$,

where $a_{k}$ is the $k$ th element of $\mathbf{a}$.

\subsection{Problem formulation}

Suppose that one of $m$ signals $\left\{s_{k}(t), 1 \leqslant k \leqslant m\right\}$ is received over an additive noise channel with equal probability, where the signals lie in a real Hilbert space $\mathscr{H}$ with inner product $\langle x(t), y(t)\rangle=\int_{t=-\infty}^{\infty} x(t) y(t) \mathrm{d} t$, and span a subspace $\mathscr{U} \subseteq \mathscr{H}$. We assume that the signals are normalized ${ }^{3}$ so that $\int_{t=-\infty}^{\infty} s_{k}^{2}(t) \mathrm{d} t=1$ for all $k$. The received signal $r(t)$ is also assumed to be in $\mathscr{H}$, and is modelled as $r(t)=s_{k}(t)+n(t)$ for one value $k$, where $n(t)$ is a stationary white noise process with zero mean and spectral density $\sigma^{2}$, and with otherwise unknown distribution.

The receiver we design consists of the correlation demodulator depicted in Fig. 1, that cross-correlates the received signal $r(t)$ with each of the $m$ signals $\left\{q_{k}(t) \in \mathscr{U}, 1 \leqslant k \leqslant m\right\}$ so that $a_{k}=\left\langle q_{k}(t), r(t)\right\rangle$, where the signals $\left\{q_{k}(t)\right\}$ are to be determined. The declared detected signal is $s_{i}(t)$ where $i=\arg \max a_{k}$. (We can equivalently obtain $a_{k}$ by filtering $r(t)$ using a filter with impulse response given by $q_{k}(-t)$, and sampling the output at $t=0$.) The essential difference

\footnotetext{
${ }^{3}$ The normalization assumption as well as the assumption that the signals are transmitted with equal probability is for notational convenience only. As we discuss in Section 8, the results readily extend to the more general case of unequal norms and unequal probabilities.
}

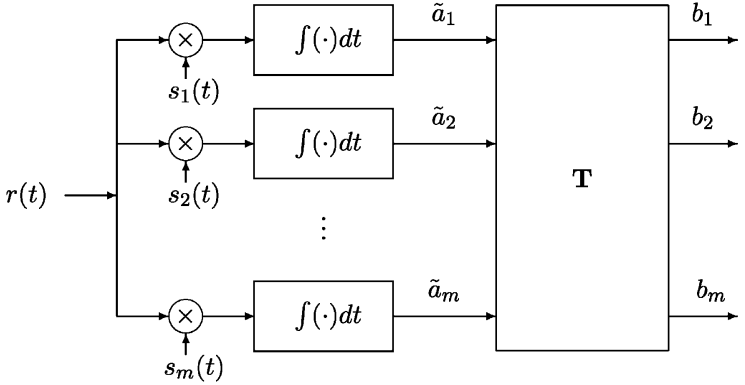

Fig. 2. Equivalent representation of a correlation demodulator. The linear transformation $\mathbf{T}$ is a function of the transmitted signals $s_{k}(t)$ and the correlating signals $q_{k}(t)$ of Fig. 1.

between the OMF and POMF receivers proposed in this paper and the MF receiver lies in the choice of the signals $q_{k}(t)$.

If we choose the signals $q_{k}(t)=s_{k}(t)$ in Fig. 1, then the resulting demodulator is equivalent to the MF demodulator [26]. If the noise is not Gaussian, then the MF receiver does not necessarily minimize the probability of a detection error. However, it is still used as the receiver of choice in many applications since the optimal receiver for non-Gaussian noise is typically nonlinear, and requires knowledge of the noise distribution.

In this paper we propose a class of correlation receivers that, like the MF receiver, depend only on the transmitted signals, and that can lead to improved performance over the MF for some classes of non-Gaussian noise, with essentially negligible loss of performance for Gaussian noise. We develop two mathematically equivalent forms of these receivers with different implications in terms of implementation. Specifically, since the signals $s_{k}(t)$ span $\mathscr{U}$ it is straightforward to show that a correlation demodulator as depicted in Fig. 1 with signals $q_{k}(t) \in \mathscr{U}$ is equivalent to a MF demodulator followed by a linear transformation $\mathbf{T}$ on the MF outputs, as depicted in Fig. 2, where the transformation $\mathbf{T}$ is constructed from the signals $s_{k}(t)$ and the correlating signals $q_{k}(t)$ of Fig. 1. The representation of Fig. 2 is of interest, since in many practical receivers the MF demodulator serves as a front-end whose objective is to obtain a vector representation of the received signal. Thus, in many applications we do not have control over the correlating signals of the correlation demodulator, but 
rather we are given the MF outputs. We may then choose to process these outputs prior to detection. If we restrict our attention to linear processing, then the overall demodulator may be expressed as a linear transformation $\mathbf{T}$ of the MF outputs, which results in a demodulator of the form depicted in Fig. 2. We first consider this representation of the correlation demodulator.

For a correlation demodulator in the form of Fig. 2, we would like to choose the transformation $\mathbf{T}$ so that when the noise is non-Gaussian the resulting detector leads to improved performance over MF detection. In the remainder of this section and in Sections 3 and 4 we focus on methods for choosing the transformation T in Fig. 2. In Sections 5 and 6 we address the design problem associated with the correlation demodulator of Fig. 1, i.e., we consider methods for choosing the signals $q_{k}(t)$.

In the system of Fig. 2, if the signals $s_{k}(t)$ are not orthonormal, then the outputs $\tilde{a}_{k}$ of the MF demodulator are correlated:

$$
\begin{aligned}
\operatorname{cov}\left(\tilde{a}_{k}, \tilde{a}_{l}\right) & =E\left(\left\langle s_{k}(t), n(t)\right\rangle\left\langle n(t), s_{l}(t)\right\rangle\right) \\
& =\sigma^{2}\left\langle s_{k}(t), s_{l}(t)\right\rangle .
\end{aligned}
$$

Intuitively it seems plausible that eliminating this common (linear) information may potentially improve the performance of the detector. Therefore, in our modification of the MF demodulator, we propose decorrelating the outputs prior to detection. The simulations in Section 7 show that when the noise is non-Gaussian this approach can in fact lead to improved performance over conventional MF detection.

Data whitening arises in a variety of signal processing and communication contexts in which it may be useful to decorrelate a data sequence either prior to subsequent processing, or to control the spectral shape after processing. Examples in which data whitening has been used to advantage include enhancing direction of arrival algorithms by pre-whitening [18,25]. Rather than choosing the whitening transformation arbitrarily, below we suggest choosing an optimal whitening transformation that whitens the MF output and at the same time minimizes the distortion to the unwhitened vector.

When the signals $s_{k}(t)$ are linearly independent, we may decorrelate the outputs of the MF demodulator by choosing $\mathbf{T}$ in Fig. 2 to be a whitening transformation
$\mathbf{T}=\mathbf{W}$. Specifically, let $\tilde{\mathbf{a}}$ denote the vector output of the MF demodulator, with components $\tilde{a}_{k}$. We propose whitening ${ }^{4} \tilde{\mathbf{a}}$ using a whitening transformation $\mathbf{T}=\mathbf{W}$ to obtain the random vector $\mathbf{b}=\mathbf{W} \mathbf{a}$, where the covariance matrix of $\mathbf{b}$ is given by $\mathbf{C}_{b}=\sigma^{2} \mathbf{I}_{m}$. The detection is then based on the components $b_{k}$ of $\mathbf{b}$, so that the declared detected signal is $s_{i}(t)$ if $i=\arg \max b_{k}$. Since the detector bases its decision on $\mathbf{b}$, we choose $\mathbf{a}$ whitening transformation $\mathbf{W}$ that minimizes the total MSE given by

$$
\begin{aligned}
\varepsilon_{\mathrm{MSE}} & =\sum_{k=1}^{m} \operatorname{var}\left(\tilde{a}_{k}-b_{k}\right)=\sum_{k=1}^{m} E\left(\left(\tilde{a}_{k}^{\prime}-b_{k}^{\prime}\right)^{2}\right) \\
& =E\left(\left(\tilde{\mathbf{a}}^{\prime}-\mathbf{b}^{\prime}\right)^{*}\left(\tilde{\mathbf{a}}^{\prime}-\mathbf{b}^{\prime}\right)\right),
\end{aligned}
$$

where $\tilde{a}_{k}^{\prime}$ and $b_{k}^{\prime}$ are the $k$ th components of $\tilde{\mathbf{a}}^{\prime}=\tilde{\mathbf{a}}-$ $E(\tilde{\mathbf{a}})$ and $\mathbf{b}^{\prime}=\mathbf{b}-E(\mathbf{b})$, respectively. That is, from all possible whitening transformations we seek the one that results in a white vector $\mathbf{b}$ that is as close as possible in a MSE sense to the output ã of the MF demodulator.

We refer to the demodulator of Fig. 2 with $\mathbf{T}=$ W chosen as the minimum MSE (MMSE) whitening transformation as the orthogonal matched filter (OMF) demodulator. This choice of terminology will become clear in Section 5, where we show that this demodulator is equivalent to a correlation demodulator matched to a set of orthogonal signals.

If the signals $s_{k}(t)$ are linearly dependent, then the outputs $\tilde{a}_{k}$ of the MF demodulator are (deterministically) linearly dependent. In this case, the vector output $\tilde{\mathbf{a}}$ of the MF demodulator lies in a subspace $\mathscr{V}$ of $\mathscr{R}^{m}$. Since the elements of a are linearly dependent, the elements of $\mathbf{b}=\mathbf{W} \tilde{\mathbf{a}}$ are also linearly dependent and therefore cannot be statistically uncorrelated, so that in this case we cannot whiten $\tilde{\mathbf{a}}$ in the conventional sense. Instead, we propose whitening ã on the subspace $\mathscr{V}$ in which it lies, which is referred to as subspace whitening [15]. The resulting demodulator has the form depicted in Fig. 2, where $\mathbf{T}=\mathbf{W}_{s}$ is a subspace whitening transformation. As in the linearly independent case, we use a subspace whitening transformation $\mathbf{W}_{s}$ such that (4) is minimized.

We refer to the demodulator of Fig. 2 with $\mathbf{T}=$ $\mathbf{W}_{s}$ chosen as the MMSE subspace whitening trans-

\footnotetext{
${ }^{4}$ In this paper we define a random vector a to be white if the covariance of $\mathbf{a}$, denoted $\mathbf{C}_{a}$, is given by $\mathbf{C}_{a}=c^{2} \mathbf{I}$ for some $c>0$.
} 
formation as the projected orthogonal matched filter (POMF) demodulator. This choice of terminology is based on the discussion in Section 5, where we show that this demodulator is equivalent to a correlation demodulator matched to a set of signals that form what we define as a projected orthogonal basis (POB) for the space spanned by the transmitted signals.

\subsection{Summary of the main results}

1. In Sections 3 and 4 we specify the OMF and POMF demodulators in terms of the implementation of Fig. 2.

2. In Section 5 we show that:

(a) The OMF demodulator is equivalent to a correlation demodulator for which the signals $q_{k}(t)$ are chosen as a set of orthonormal signals $\hat{g}_{k}(t)$ that are closest to the transmitted signals $s_{k}(t)$ in a least-squares sense (Section 5.1).

(b) The POMF demodulator is equivalent to a correlation demodulator with signals $\hat{f}_{k}(t)$ that form a POB for the space spanned by the transmitted signals, and is closest to the signals $s_{k}(t)$ in a least-squares sense (Section 5.2).

(c) The OMF and POMF signals may equivalently be obtained by maximizing the sum of the inner products $R_{k}=\left\langle q_{k}(t), s_{k}(t)\right\rangle$, subject to the constraint that the outputs of the demodulator are uncorrelated on $\mathscr{V}$ (Sections 5.1 and 5.2).

3. In Section 6 we show that when the transmitted signals have a symmetry property that is common to most signal sets used in digital communication [17], the OMF and POMF demodulators maximize the total output SNR subject to the decorrelation constraint.

\section{The orthogonal matched filter demodulator}

To specify the OMF demodulator for the case in which the signals $\left\{s_{k}(t), 1 \leqslant k \leqslant m\right\}$ are linearly independent, we need to specify the MMSE whitening transformation $\mathbf{T}=\mathbf{W}$ of Fig. 2. The solution to the general MMSE whitening problem has been obtained in $[13,15]$, and is incorporated in the following theorem:
Theorem 1 (MMSE whitening transformation $[13,15])$. Let $\mathbf{z} \in \mathscr{R}^{m}$ be a zero-mean random vector with positive-definite covariance matrix $\mathbf{C}_{z}$ with eigendecomposition $\mathbf{C}_{z}=\mathbf{U D U}{ }^{*}$, where $\mathbf{U}$ is a unitary matrix and $\mathbf{D}$ is a diagonal matrix. Among all possible whitening transformations, let $\hat{\mathbf{W}}$ denote the whitening transformation that minimizes the total $M S E$,

$\varepsilon_{\mathrm{MSE}}=\sum_{k=1}^{m} E\left(\left(z_{k}-h_{k}\right)^{2}\right)=E\left((\mathbf{z}-\mathbf{h})^{*}(\mathbf{z}-\mathbf{h})\right)$,

between the input $\mathbf{z}$ with elements $z_{k}$ and the output $\mathbf{h}=\mathbf{W z}$ with covariance $\mathbf{C}_{h}=c^{2} \mathbf{I}_{m}, c>0$, and elements $h_{m}$. Then

$\hat{\mathbf{W}}=c \mathbf{U D}^{-1 / 2} \mathbf{U}^{*}=c \mathbf{C}_{z}^{-1 / 2}$.

Now, let $S: \mathscr{R}^{m} \rightarrow \mathscr{H}$ be the set transformation corresponding to the signals $s_{k}(t)$. From (3) it follows that the covariance matrix of the input a to the whitening transformation of Fig. 2 is given by

$\mathbf{C}_{a}=\sigma^{2} S^{*} S$,

where the $k l$ th element of $S^{*} S$ is $\left\langle s_{k}(t), s_{l}(t)\right\rangle$, and the covariance of the output $\mathbf{b}$ is $\mathbf{C}_{b}=\sigma^{2} \mathbf{I}_{m}$. The MMSE whitening transformation then follows from Theorem 1,

$\hat{\mathbf{W}}=\left(S^{*} S\right)^{-1 / 2}$.

Thus, the OMF demodulator consists of a MF demodulator followed by the optimal whitening transformation given by (6).

\section{The projected orthogonal matched filter demodulator}

We now consider the case in which the transmitted signals $\left\{s_{k}(t), 1 \leqslant k \leqslant m\right\}$ are linearly dependent, and span an $n$-dimensional subspace $\mathscr{U} \subset \mathscr{H}$, where $n<m$.

As in the linearly independent case, we would like to whiten the output $\mathbf{a}$ of the MF demodulator prior to detection. However since the signals $s_{k}(t)$ are linearly dependent, the components of $\tilde{\mathbf{a}}$ and consequently of $\mathbf{b}=\mathbf{W} \tilde{\mathbf{a}}$, are linearly dependent and therefore cannot be statistically uncorrelated. Equivalently, the covariance matrix $\mathbf{C}_{a}=\sigma^{2} S^{*} S$ of $\tilde{\mathbf{a}}$ is not invertible and therefore there is no whitening transformation $\mathbf{W}$ 
such that $\mathbf{W C}_{a} \mathbf{W}^{*}=\sigma^{2} \mathbf{I}_{m}$. Instead, we propose whitening $\tilde{\mathbf{a}}$ on the space in which it is contained. Specifically, let $\mathscr{V} \subset \mathscr{R}^{m}$ denote the range space of $S^{*}$, which from (5) is equal to the range space of $\mathbf{C}_{a}$. Then we propose whitening $\tilde{\mathbf{a}}$ on the subspace $\mathscr{V}$, which is referred to as subspace whitening [15]. The output of the subspace transformation $\mathbf{b}$ is said to be white on $\mathscr{V}$ if its covariance matrix $\mathbf{C}_{b}$ is given by

$\mathbf{C}_{b}=\sigma^{2} P_{\mathscr{V}}=\sigma^{2} \mathbf{V} \tilde{\mathbf{I}} \mathbf{V}^{*}$

where the first $n$ columns of $\mathbf{V}$ form an orthonormal basis for $\mathscr{V}$, and $\tilde{\mathbf{I}}$ is the $m \times m$ matrix

$\tilde{\mathbf{I}}=\left[\begin{array}{ll}\mathbf{I}_{n} & 0 \\ 0 & 0\end{array}\right]$.

Condition (7) implies that the representation of $\mathbf{b}$ in terms of any orthonormal basis for $\mathscr{V}$ is white in the conventional sense. A more detailed discussion on subspace whitening can be found in [15].

The solution to the general MMSE subspace whitening problem has also been obtained in [15], and is incorporated in the following theorem:

Theorem 2 (MMSE subspace whitening [15]). Let $\mathbf{z} \in \mathscr{R}^{m}$ be a zero mean random vector with covariance matrix $\mathbf{C}_{z}=\mathbf{U D U}^{*}$, with $\operatorname{rank}\left(\mathbf{C}_{z}\right)=n<m$. Let $\mathscr{V}$ denote the range space of $\mathbf{C}_{z}$. Let $\hat{\mathbf{W}}_{s}$ be any subspace whitening transformation that minimizes the total MSE between the input $\mathbf{z}$ and the output $\mathbf{h}$ with covariance $\mathbf{C}_{h}=c^{2} P_{\mathscr{V}}=c^{2} \mathbf{U} \tilde{\mathbf{I}}{ }^{*}$, where $\tilde{\mathbf{I}}$ is given by (8) and $c>0$. Then

1. $\hat{\mathbf{W}}_{s}$ is not unique;

2. $\hat{\mathbf{W}}_{s}=c \mathbf{U}\left(\mathbf{D}^{1 / 2}\right)^{\dagger} \mathbf{U}^{*}=c\left(\mathbf{C}_{z}^{1 / 2}\right)^{\dagger}$ is an optimal subspace whitening transformation.

Furthermore, let $\mathbf{W}_{s}^{\mathscr{V}}=\hat{\mathbf{W}}_{s} P_{\mathscr{V}}$ where $P_{\mathscr{V}}$ is an orthogonal projection onto $\mathscr{V}$ and $\hat{\mathbf{W}}_{s}$ is any optimal subspace whitening transformation. Then

1. $\mathbf{W}_{s}^{\mathscr{V}}$ is unique, and is given by $\mathbf{W}_{s}^{\mathscr{V}}=c \mathbf{U}\left(\mathbf{D}^{1 / 2}\right)^{\dagger} \mathbf{U}^{*}=$ $c\left(\mathbf{C}_{z}^{1 / 2}\right)^{\dagger}$

2. $\hat{\mathbf{W}}_{s} \mathbf{z}=\mathbf{W}_{s}^{\mathscr{V}} \mathbf{z}$ w.p. 1;

3. $\mathbf{h}=\hat{\mathbf{W}}_{s} \mathbf{z}$ is unique w.p. 1 .

In Fig. 2 the input to the whitening transformation $\mathbf{T}=\mathbf{W}_{s}$ is $\tilde{\mathbf{a}}=S^{*} r(t)$ with $\mathbf{C}_{a}=\sigma^{2} S^{*} S$. From Theorem 2 the optimal subspace whitening transformation in this case is

$\hat{\mathbf{W}}_{s}=\left(\left(S^{*} S\right)^{1 / 2}\right)^{\dagger}$.

Thus, the POMF demodulator consists of a MF demodulator followed by an optimal subspace whitening transformation given by (9).

Note, that in Fig. 2 a is restricted to the range space $\mathscr{V}$ of $\mathbf{C}_{a}$, since $\mathscr{V}$ is also the range of $S^{*}$ and $\tilde{\mathbf{a}}=S^{*} r(t)$. Consequently the output $\mathbf{b}$ is not affected by the action of $\hat{\mathbf{W}}_{s}$ on $\mathscr{V}^{\perp}$.

\section{Correlation demodulator representation of the OMF and POMF demodulators}

\subsection{Correlation demodulator representation of the OMF demodulator}

The output of Fig. 2 can be expressed as $\mathbf{b}=\mathbf{T} S^{*} r(t)=Q^{*} r(t)$ where $Q=S \mathbf{T}^{*}$. Therefore, a demodulator of the form depicted in Fig. 2 is equivalent to a correlation demodulator with correlating signals $q_{k}(t)=Q \mathbf{i}_{k}=S \mathbf{T}^{*} \mathbf{i}_{k}$, where the $l$ th component of $\mathbf{i}_{k}$ is $\delta_{k l}$. If $\mathbf{T}$ in Fig. 2 is a whitening transformation $\mathbf{W}$, then the demodulator is equivalent to a correlation demodulator with signals denoted by $g_{k}(t)$ corresponding to $G=S \mathbf{W}^{*}$. Since $\mathbf{C}_{a}=\sigma^{2} S^{*} S$, W must satisfy $\mathbf{W} S^{*} S \mathbf{W}^{*}=\mathbf{I}_{m}$, so that

$G^{*} G=\mathbf{W} S^{*} S \mathbf{W}^{*}=\mathbf{I}_{m}$.

In Appendix A we show that a set transformation $G$ corresponding to signals $g_{k}(t)$ satisfies (10) if and only if the signals $g_{k}(t)$ are orthonormal. We therefore conclude that a MF demodulator followed by a whitening transformation is equivalent to a correlation demodulator with orthonormal signals $g_{k}(t)$.

\subsubsection{OMF signals}

The OMF demodulator consists of a MF demodulator followed by the MMSE whitening transformation $\hat{\mathbf{W}}$ given by (6), and is therefore equivalent to a correlation demodulator with orthonormal signals $q_{k}(t)=\hat{g}_{k}(t)$, where $\hat{g}_{k}(t)=\hat{G} \mathbf{i}_{k}$ and

$\hat{G}=S \hat{\mathbf{W}}^{*}=S\left(S^{*} S\right)^{-1 / 2}$.

We refer to the signals $\hat{g}_{k}(t)$ as the OMF signals.

Since every whitening transformation defines a set of orthonormal correlating signals, and the MMSE 
transformation is optimal in some sense, we expect the OMF signals to also have some form of optimality. Indeed, we now show that the OMF signals are the closest orthonormal signals to the signals $s_{k}(t)$ in a least-squares sense.

Let $b_{k}$ denote the $k$ th output of the demodulator of Fig. 2, where $\mathbf{T}=\mathbf{W}$ is an arbitrary whitening transformation. Then $b_{k}=\left\langle g_{k}(t), r(t)\right\rangle$, where $g_{k}(t)$ are the orthonormal signals corresponding to the set transformation $G=S \mathbf{W}^{*}$. Let $\tilde{a}_{k}=\left\langle s_{k}(t), r(t)\right\rangle$ denote the $k$ th output of the MF demodulator. Then, $\tilde{a}_{k}^{\prime}=\left\langle s_{k}(t), n(t)\right\rangle, b_{k}^{\prime}=\left\langle g_{k}(t), n(t)\right\rangle$ and

$$
\begin{aligned}
& E\left(\left(\tilde{a}_{k}^{\prime}-b_{k}^{\prime}\right)^{2}\right) \\
& =E\left(\left\langle s_{k}(t)-g_{k}(t), n(t)\right\rangle\left\langle n(t), s_{k}(t)-g_{k}(t)\right\rangle\right) \\
& =E\left(\left(\int_{t=-\infty}^{\infty}\left(s_{k}(t)-g_{k}(t)\right) n(t) \mathrm{d} t\right)^{2}\right) \\
& =\sigma^{2} \int_{t=-\infty}^{\infty}\left(s_{k}(t)-g_{k}(t)\right)^{2} \mathrm{~d} t \\
& =\sigma^{2}\left\langle s_{k}(t)-g_{k}(t), s_{k}(t)-g_{k}(t)\right\rangle .
\end{aligned}
$$

From (12) we conclude that minimizing the MSE defined by (4) is equivalent to minimizing the least-squares error $\varepsilon_{\mathrm{LS}}\left(\left\{s_{k}(t)\right\},\left\{g_{k}(t)\right\}\right)$, where

$$
\varepsilon_{\mathrm{LS}}\left(\left\{s_{k}(t)\right\},\left\{g_{k}(t)\right\}\right)
$$

$$
=\sum_{k=1}^{m}\left\langle s_{k}(t)-g_{k}(t), s_{k}(t)-g_{k}(t)\right\rangle \text {. }
$$

Thus, seeking a whitening transformation to minimize the MSE defined by (4) is equivalent to seeking a set of orthonormal vectors $g_{k}(t)$ that are closest to the signals $s_{k}(t)$ in a least-squares sense. This problem has also been solved in the context of quantum detection [9] and in the context of general inner product shaping [6]; the solution in [6,9] is equal to the OMF signals corresponding to the set transformation given by (11). We may then interpret the OMF demodulator as a correlation demodulator matched to a set of orthonormal signals that are closest in the least-squares sense to the signals $s_{k}(t)$. These closest orthonormal signals are the OMF signals.

An alternative method for designing the OMF signals that does not make use of the equivalence between the correlation demodulator and the demodulator of Fig. 2 is developed in [13]. This method is based on the observation that if the transmitted signal is $s_{i}(t)$, then in Fig. $1, a_{k}=\left\langle g_{k}(t), r(t)\right\rangle$ and the declared detected signal will be the transmitted signal if $\max _{k}\left\langle g_{k}(t), s_{i}(t)+n(t)\right\rangle=\left\langle g_{i}(t), s_{i}(t)+n(t)\right\rangle$. Therefore a reasonable approach is to choose the signals $g_{k}(t)$ to be orthonormal, so that the outputs $a_{k}$ are uncorrelated, and to maximize the sum of the inner products,

$R\left(\left\{g_{k}(t)\right\},\left\{s_{k}(t)\right\}\right)=\sum_{k=1}^{m}\left\langle g_{k}(t), s_{k}(t)\right\rangle$.

Since $\left\langle g_{k}(t), g_{k}(t)\right\rangle=1$ independent of the choice of signals $g_{k}(t)$, maximizing $R\left(\left\{g_{k}(t)\right\},\left\{s_{k}(t)\right\}\right)$ is equivalent to minimizing the least-squares error $\varepsilon_{\mathrm{LS}}\left(\left\{s_{k}(t)\right\},\left\{g_{k}(t)\right\}\right)$ defined by (13). Thus, the orthonormal signals $g_{k}(t)$ that maximize (14) are equal to the OMF signals.

\subsection{Correlation demodulator representation of the POMF demodulator}

If $\mathbf{T}=\mathbf{W}_{s}$ is a subspace whitening transformation on the range space $\mathscr{V}$ of $S^{*}$, then in a manner similar to the discussion in Section 5.1 it follows that the corresponding correlating signals are the signals denoted $f_{k}(t)$ corresponding to $F$, where $F=S \mathbf{W}_{s}^{*}$. Since $\mathbf{W}_{s}$ is a subspace whitening transformation on $\mathscr{V}, \mathbf{W}_{s} S^{*} S \mathbf{W}_{s}^{*}=P_{\mathscr{V}}$, and

$F^{*} F=\mathbf{W}_{s} S^{*} S \mathbf{W}_{s}^{*}=P_{\mathscr{V}}$.

In Section 5.2.1 we define a projected orthogonal basis (POB) and show that although the signals $f_{k}(t)$ are not orthonormal, (15) implies that they form a POB. From this we can then conclude that a MF demodulator followed by a subspace whitening transformation is equivalent to a correlation demodulator with signals that form a POB.

\subsubsection{Projected orthogonal basis}

Let $\mathscr{U}$ be an $n$-dimensional subspace of $\mathscr{H}$, and let $\mathscr{M}$ be an $m$-dimensional subspace of $\mathscr{H}$ containing $\mathscr{U}$, so that $m>n$. Suppose we project a set of signals $\left\{x_{k}(t), 1 \leqslant k \leqslant m\right\}$ which are orthonormal in the larger space $\mathscr{M}$ onto the smaller space $\mathscr{U}$, to form the projections

$z_{k}(t)=P_{\mathscr{U}} x_{k}(t), \quad 1 \leqslant k \leqslant m$. 
With $X$ and $Z$ denoting the set transformations corresponding to the signals $x_{k}(t)$ and $z_{k}(t)$ respectively, it follows from (16) that $Z=P_{\mathscr{U}} X$. Since the vectors $x_{k}(t)$ form an orthonormal basis for $\mathscr{M}, X X^{*}=P_{\mathscr{M}}$, and

$Z Z^{*}=P_{\mathscr{U}} X X^{*} P_{\mathscr{U}}=P_{\mathscr{U}} P_{\mathscr{M}} P_{\mathscr{U}}=P_{\mathscr{U}}$,

where the last equality follows from the fact that $\mathscr{U} \subset \mathscr{M}$. Equivalently, for any $y(t) \in \mathscr{U}$ we have

$y(t)=P_{\mathscr{U}} y(t)=Z Z^{*} y(t)=\sum_{k=1}^{m}\left\langle z_{k}(t), y(t)\right\rangle z_{k}(t)$.

The expansion of (18) is reminiscent of a basis expansion of $y(t)$ in terms of an orthonormal basis for $\mathscr{U}$. However, while the signals in an expansion in an orthonormal basis are linearly independent, the $m>n$ signals $z_{k}(t)$ in (18) are linearly dependent and consequently do not form a basis for $\mathscr{U}$. Instead, they are defined as a POB for $\mathscr{U}$ since they span $\mathscr{U}$ and are projections of an orthonormal basis onto $\mathscr{U}$.

We therefore have the following definition: The signals $\left\{z_{k}(t) \in \mathscr{U}, 1 \leqslant k \leqslant m\right\}$ form a projected orthogonal basis $(P O B)$ for an $n$-dimensional space $\mathscr{U}$, with $n<m$, if

$Z Z^{*}=P_{\mathscr{U}}$,

where $Z$ is the set transformation corresponding to the signals $z_{k}(t)$.

The signals $\left\{z_{k}(t) \in \mathscr{U}, 1 \leqslant k \leqslant m\right\}$ form a scaled $P O B$ for $\mathscr{U}$ if $Z Z^{*}=c P_{\mathscr{U}}$ for some $c>0$.

We note that a POB for $\mathscr{U}$ constitutes a tight frame $[1,4]$ for $\mathscr{U}$; furthermore, any tight frame for $\mathscr{U}$ is a scaled POB for $\mathscr{U}[10]$. Thus, a POB is a tight frame with a normalization constraint. We prefer the nomenclature POB since frames are typically associated with redundant expansions, while in this paper scaled tight frames arise more naturally as projections of orthogonal bases; the redundant expansion property of tight frames is not relevant to our presentation.

It can be shown that any POB for $\mathscr{U}$ can be expressed as the projection of a set of $m$ orthonormal signals in an $m$-dimensional space $\mathscr{M}$ onto $\mathscr{U}$, where $\mathscr{U} \in \mathscr{M}$ [10]. Thus, if $Z$ satisfies (19) then there is some set transformation $X$ corresponding to a set of orthonormal signals such that $Z=P_{\mathscr{U}} X$. It then follows in a straightforward way that $Z^{*} Z=X^{*} P_{\mathscr{U}} X$ is a projection operator. Indeed, $X^{*} P_{\mathscr{U}} X X^{*} P_{\mathscr{U}} X=$
$X^{*} P_{\mathscr{U}} P_{\mathscr{M}} P_{\mathscr{U}} X=X^{*} P_{\mathscr{U}} X$. Thus any set transformation $Z$ that satisfies (19) also satisfies

$Z^{*} Z=P_{Z^{*}}$,

where $P_{Z^{*}}$ is an orthogonal projection onto the range of $Z^{*}$, so that the signals $\left\{z_{k}(t) \in \mathscr{U}, 1 \leqslant k \leqslant m\right\}$ form a POB for the space they span if and only if $Z^{*} Z$ is a projection operator.

Comparing (15) with (20) we conclude that the signals $f_{k}(t)$ corresponding to a set transformation $F$ that satisfies (15), forms a POB for the space they span.

\subsubsection{POMF signals}

The POMF demodulator is equivalent to a correlation demodulator with signals that form a POB, and are given by $\hat{f}_{k}(t)=\hat{F} \mathbf{i}_{k}$ where

$\hat{F}=S \hat{\mathbf{W}}_{s}^{*}=S\left(\left(S^{*} S\right)^{1 / 2}\right)^{\dagger}$,

and $\hat{\mathbf{W}}_{s}$ is the optimal MMSE whitening transformation given by (9). We refer to the signals $\hat{f}_{k}(t)$ as the POMF signals. Since the signals $\hat{f}_{k}(t)$ span $\mathscr{U}$ (the space spanned by the transmitted signals), the POMF demodulator is equivalent to a correlation demodulator with signals $q_{k}(t)=\hat{f}_{k}(t)$ that form a POB for $\mathscr{U}$.

If $\mathbf{T}=\mathbf{W}_{s}$ is an arbitrary subspace whitening transformation in the system of Fig. 2, then $b_{k}=\left\langle f_{k}(t), r(t)\right\rangle$, where $f_{k}(t)$ is the POB corresponding to the set transformation $F=S \mathbf{W}_{s}$. Substituting $f_{k}(t)$ for $g_{k}(t)$ in (12) we conclude that the signals $\hat{f}_{k}(t)$ minimize the least-squares error $\varepsilon_{\mathrm{LS}}\left(\left\{s_{k}(t)\right\},\left\{f_{k}(t)\right\}\right)$. Thus, the POMF demodulator may be interpreted as a correlation demodulator with signals $\hat{f}_{k}(t)$ that form a POB that is closest in a least-squares sense to the signals $s_{k}(t)$.

As in the case of linearly independent signals, we may derive the POMF signals by seeking a set of signals $\left\{f_{k}(t), 1 \leqslant k \leqslant m\right\}$ that form a POB and such that $R\left(\left\{f_{k}(t)\right\},\left\{s_{k}(t)\right\}\right)$, defined by (14), is maximized. Note that contrary to the linearly independent case, here $\left\langle f_{k}(t), f_{k}(t)\right\rangle$ depends on the choice of $f_{k}(t)$. In Appendix $\mathrm{B}$ we show that maximizing $R\left(\left\{f_{k}(t)\right\},\left\{s_{k}(t)\right\}\right)$ subject to the constraint that the signals $f_{k}(t)$ form a POB, is equivalent to minimizing $\varepsilon_{\mathrm{LS}}\left(\left\{s_{k}(t)\right\},\left\{f_{k}(t)\right\}\right)$ subject to this constraint. Therefore, the POB signals $f_{k}(t)$ that maximize $R\left(\left\{f_{k}(t)\right\},\left\{s_{k}(t)\right\}\right)$ are equal to the POMF signals. 


\subsection{Matrix representation of the $O M F$ and $P O M F$ signals}

To implement the OMF and POMF demodulators in practice, we may find it more convenient to reformulate the OMF and POMF signals in terms of their coefficients in a basis expansion for the space $\mathscr{U}$ spanned by the transmitted signals. These coefficients can be viewed as vectors in a coefficient space $\mathscr{R}^{p}$, with $p=m$ for the OMF signals and $p=n$ for the POMF signals.

\subsubsection{Matrix representation of the OMF signals}

Let $\mathscr{U}$ denote the $m$-dimensional subspace spanned by the signals $s_{k}(t)$, and let $X: \mathscr{R}^{m} \rightarrow \mathscr{U}$ denote a set transformation corresponding to a set of $m$ signals that form an orthonormal basis for $\mathscr{U}$. Then $s_{k}(t)=X \mathbf{s}_{k}$ for some $\mathbf{s}_{k} \in \mathscr{R}^{m}$, and $S=X \mathbf{S}$ where $\mathbf{S}$ is the $m \times m$ matrix of columns $\mathbf{s}_{k}$. We may then express $\hat{G}$ of (11) in terms of $X$ and $\mathbf{S}$ as

$\hat{G}=S\left(S^{*} S\right)^{-1 / 2}=X \mathbf{S}\left(\mathbf{S}^{*} \mathbf{S}\right)^{-1 / 2}$,

where we used the fact that $X^{*} X=I_{m}$. Thus, $\hat{g}_{k}(t)=$ $X \hat{\mathbf{g}}_{k}$ where $\hat{\mathbf{g}}_{k}$ is the $k$ th column of the $m \times m$ matrix $\hat{\mathbf{G}}$, and

$\hat{\mathbf{G}}=\mathbf{S}\left(\mathbf{S}^{*} \mathbf{S}\right)^{-1 / 2}$.

Since (23) has the same form as (22), we conclude that the vectors $\left\{\hat{\mathbf{g}}_{k}, 1 \leqslant k \leqslant m\right\}$ are the closest orthonormal vectors to the vectors $\left\{\mathbf{s}_{k}, 1 \leqslant k \leqslant m\right\}$, in the least-squares sense. We may therefore determine the OMF signals by solving the least-squares problem of (13) in the coefficient space $\mathscr{R}^{m}$.

We may express $\hat{\mathbf{G}}$ in terms of the polar decomposition [22] and the singular value decomposition (SVD) [19] of S. Rearranging (23) we have

$\mathbf{S}=\hat{\mathbf{G}}\left(\mathbf{S}^{*} \mathbf{S}\right)^{1 / 2}=\hat{\mathbf{G}} \mathbf{A}$,

where $\hat{\mathbf{G}}$ is a partial isometry ${ }^{5}$ and $\mathbf{A}$ is a symmetric positive-definite matrix, so that (24) is the polar decomposition of $\mathbf{S}$. Thus, $\hat{\mathbf{G}}$ is just the partial isometry in this decomposition. Next, let the SVD of $\mathbf{S}$ be given by $\mathbf{S}=\mathbf{U} \boldsymbol{\Sigma} \mathbf{V}^{*}$, where $\mathbf{U}$ and $\mathbf{V}$ are unitary matrices

\footnotetext{
${ }^{5}$ A partial isometry is a matrix $\mathbf{H}$ that satisfies $\mathbf{H}^{*} \mathbf{H}=\mathbf{I}$, where $\mathbf{H}$ is not necessarily a square matrix.
}

and $\boldsymbol{\Sigma}$ is diagonal. Substituting into (23) we have $\hat{\mathbf{G}}=\mathbf{U} \mathbf{V}^{*}$.

Using (24) and (25), the OMF signals may be computed very efficiently exploiting the many known efficient algorithms for computing the polar decomposition and the SVD (see, e.g., $[19,21,29]$ ).

\subsubsection{Matrix representation of the POMF signals}

If the signals $s_{k}(t)$ are linearly dependent, then they form an $n$-dimensional subspace $\mathscr{U}$, where $n<m$. Let $X: \mathscr{R}^{n} \rightarrow \mathscr{U}$ denote a set transformation corresponding to a set of $n$ signals that form an orthonormal basis for $\mathscr{U}$. Then $s_{k}(t)=X \mathbf{s}_{k}$ for some $s_{k} \in \mathscr{R}^{n}$, and $S=X \mathbf{S}$ where $\mathbf{S}$ is the $n \times m$ matrix of columns $\mathbf{s}_{k}$. We can now express $\hat{F}$ in terms of $X$ and $\mathbf{S}$ as

$\hat{F}=S\left(\left(S^{*} S\right)^{1 / 2}\right)^{\dagger}=X \mathbf{S}\left(\left(\mathbf{S}^{*} \mathbf{S}\right)^{1 / 2}\right)^{\dagger}$.

Thus, $\hat{f}_{k}(t)=X \hat{\mathbf{f}}_{k}$ where $\hat{\mathbf{f}}_{k}$ is the $k$ th column of the $n \times m$ matrix $\hat{\mathbf{F}}$, and

$\hat{\mathbf{F}}=\mathbf{S}\left(\left(\mathbf{S}^{*} \mathbf{S}\right)^{1 / 2}\right)^{\dagger}$.

Since (27) has the same form as (26), we conclude that the vectors $\left\{\hat{\mathbf{f}}_{k}, 1 \leqslant k \leqslant m\right\}$ form the closest POB to the vectors $\left\{\mathbf{s}_{k}, 1 \leqslant k \leqslant m\right\}$, in the least-squares sense. We may therefore determine the POMF signals by solving the least-squares problem of (13) in the coefficient space $\mathscr{R}^{n}$, subject to the POB constraint.

We may express $\hat{\mathbf{F}}$ in terms of the SVD of $\mathbf{S}, \mathbf{S}=$ $\mathbf{U} \boldsymbol{\Sigma} \mathbf{V}^{*}$, where $\mathbf{U}$ and $\mathbf{V}$ are unitary matrices and $\boldsymbol{\Sigma}$ is diagonal, as

$\hat{\mathbf{F}}=\mathbf{U} \mathbf{I}_{n m} \mathbf{V}^{*}$,

where $\mathbf{I}_{n m}$ is the $n \times m$ matrix given by

$\mathbf{I}_{n m}=\left[\begin{array}{ll}\mathbf{I}_{n} & \mathbf{0}_{n \times(m-n)}\end{array}\right]$.

In this case it can be shown that $\hat{\mathbf{F}}$ is the projection onto the space spanned by the vectors $\mathbf{S}_{k}$ of the partial isometry in a polar decomposition ${ }^{6}$ of $\mathbf{S}$. In practice, we may find it convenient to compute the POMF signals through the use of (28).

\footnotetext{
${ }^{6}$ If the columns $\mathbf{s}_{k}$ of $\mathbf{S}$ are linearly dependent, then the partial isometry in the polar decomposition of $\mathbf{S}$ is not unique; however its projection onto the space spanned by the signals $\mathbf{s}_{k}$ is unique [6].
} 


\section{Summary of the OMF and POMF demodulators}

We summarize our results regarding the OMF and POMF demodulators in the following theorems:

Theorem 3 (OMF demodulator). Let $\left\{s_{k}(t), 1 \leqslant\right.$ $k \leqslant m\}$ denote a set of $m$ transmitted signals in a Hilbert space $\mathscr{H}$ that span an m-dimensional subspace $\mathscr{U} \subseteq \mathscr{H}$. Let $\left\{\hat{g}_{k}(t), 1 \leqslant k \leqslant m\right\}$ denote the $O M F$ signals that are the correlating signals of the OMF demodulator. Let $S: \mathscr{R}^{m} \rightarrow \mathscr{H}$ and $\hat{G}: \mathscr{R}^{m} \rightarrow$ $\mathscr{H}$ denote the set transformations corresponding to the signals $s_{k}(t)$ and $\hat{g}_{k}(t)$, respectively. Then

$\hat{G}=S\left(S^{*} S\right)^{-1 / 2}$.

Let $X$ denote a set transformation corresponding to an orthonormal basis for $\mathscr{U}$, let $s_{k}(t)=X \mathbf{s}_{k}$, and let $\mathbf{S}$ be the matrix of columns $\mathbf{s}_{k}$ with $S V D \mathbf{S}=\mathbf{U} \mathbf{\Sigma} \mathbf{V}^{*}$. Then $\hat{g}_{k}(t)=X \hat{\mathbf{g}}_{k}$ where the vectors $\hat{\mathbf{g}}_{k}$ are the columns of $\hat{\mathbf{G}}$, and

$\hat{\mathbf{G}}=\mathbf{S}\left(\mathbf{S}^{*} \mathbf{S}\right)^{-1 / 2}=\mathbf{U} \mathbf{V}^{*}$.

In addition,

1. the OMF demodulator can be realized by a conventional MF demodulator followed by the MMSE whitening transformation $\hat{\mathbf{W}}=$ $\left(S^{*} S\right)^{-1 / 2}=\left(\mathbf{S}^{*} \mathbf{S}\right)^{-1 / 2}$;

2. the signals $\hat{g}_{k}(t)$ minimize the least-squares error given by (13), i.e., they are the closest orthonormal signals to the signals $s_{k}(t)$

3. the vectors $\hat{\mathbf{g}}_{k}$ are the closest orthonormal vectors to the vectors $\mathbf{s}_{k}$, and are the columns of the partial isometry in the polar decomposition of $\mathbf{S}$;

4. the signals $\hat{g}_{k}(t)$ maximize the sum of the inner products $R\left(\left\{g_{k}(t)\right\},\left\{s_{k}(t)\right\}\right)$ given by (14).

Theorem 4 (POMF demodulator). Let $\left\{s_{k}(t), 1 \leqslant\right.$ $k \leqslant m\}$ denote a set of $m$ transmitted signals in a Hilbert space $\mathscr{H}$ that span an $n$-dimensional subspace $\mathscr{U} \subset \mathscr{H}$. Let $\left\{\hat{f}_{k}(t), 1 \leqslant k \leqslant m\right\}$ denote the $P O M F$ signals that are the correlating signals of the POMF demodulator. Let $S: \mathscr{R}^{m} \rightarrow \mathscr{H}$ and $\hat{F}: \mathscr{R}^{m} \rightarrow$ $\mathscr{H}$ denote the set transformations corresponding to the signals $s_{k}(t)$ and $\hat{f}_{k}(t)$, respectively. Then

$\hat{F}=S\left(\left(S^{*} S\right)^{1 / 2}\right)^{\dagger}$.
Let $X$ denote a set transformation corresponding to an orthonormal basis for $\mathscr{U}$, let $s_{k}(t)=X \mathbf{s}_{k}$, and let $\mathbf{S}$ be the matrix of columns $\mathbf{s}_{k}$ with $S V D \mathbf{S}=\mathbf{U} \mathbf{\Sigma} \mathbf{V}^{*}$. Then $\hat{f}_{k}(t)=X \hat{\mathbf{f}}_{k}$ where the vectors $\hat{\mathbf{f}}_{k}$ are the columns of $\hat{\mathbf{F}}$, and

$\hat{\mathbf{F}}=\mathbf{S}\left(\left(\mathbf{S}^{*} \mathbf{S}\right)^{1 / 2}\right)^{\dagger}=\mathbf{U} \mathbf{I}_{n m} \mathbf{V}^{*}$,

where $\mathbf{I}_{n m}$ is given by (29). In addition,

1. the POMF demodulator can be realized by a conventional MF demodulator followed by an optimal subspace whitening transformation $\hat{\mathbf{W}}_{s}=\left(\left(S^{*} S\right)^{1 / 2}\right)^{\dagger}=\left(\left(\mathbf{S}^{*} \mathbf{S}\right)^{1 / 2}\right)^{\dagger}$;

2. the signals $\hat{f}_{k}(t)$ minimize the least-squares error $\varepsilon_{\mathrm{LS}}\left(\left\{s_{k}(t)\right\},\left\{f_{k}(t)\right\}\right)$ given by (13), i.e., they form the closest $P O B$ to the signals $s_{k}(t)$;

3. the vectors $\hat{\mathbf{f}}_{k}$ form the closest POB to the vectors $\mathbf{s}_{k}$, and are the projections onto $\mathscr{U}$ of the columns of the partial isometry in a polar decomposition of $\mathbf{S}$;

4. the signals $\hat{f}_{k}(t)$ maximize the sum of the inner products $R\left(\left\{f_{k}(t)\right\},\left\{s_{k}(t)\right\}\right)$ given by (14).

Finally, we note that based on results derived in the context of quantum detection [9] it can be shown that in many cases the OMF and POMF demodulators have an additional property, analogous to the SNR property of the MF demodulator.

Specifically, it is well known that choosing $q_{k}(t)=s_{k}(t)$ in Fig. 1 maximizes the SNR of $a_{k}$, and also of course maximizes the total SNR defined by $\mathrm{SNR}_{T}=\left(1 / \sigma^{2}\right) \sum_{k=1}^{m}\left|\left\langle q_{k}(t), s_{k}(t)\right\rangle\right|^{2}$, since the individual terms are maximized by this choice. To design our receiver, we may therefore seek a set of signals that maximize $\mathrm{SNR}_{T}$ subject to the orthogonality constraint or the POB constraint, so as to ensure that the outputs are uncorrelated on the space in which they lie. Although this is a reasonable approach to choosing the signals $q_{k}(t)$, this problem is hard to solve analytically.

However, we can formulate this problem within the framework of quantum detection, and then rely on results obtained in that context [5]. Specifically based on the results in $[5,9,12]$, it can be shown that when the signals $s_{k}(t)$ have a strong symmetry property called geometric uniformity [17] or compound geometric uniformity [7] the OMF and POMF 
signals maximize $\mathrm{SNR}_{T}$ subject to the constraint that the outputs of the demodulator are uncorrelated on the space in which they lie. In [17] it is claimed that most practical signal sets used in digital communication are indeed geometrically uniform. Thus, in a communications context the POMF and OMF demodulators have a property analogous to the MF demodulator, namely they typically maximize the total $\mathrm{SNR}_{T}$ subject to the decorrelation constraint.

Further results regarding the orthogonal or $\mathrm{POB}$ signals that maximize $\mathrm{SNR}_{T}$ that follow from results pertaining to quantum detection are that if the signals are nearly orthogonal, then the OMF and POMF signals maximize $\mathrm{SNR}_{T}[5,20]$. Iterative algorithms for maximizing $\mathrm{SNR}_{T}$ for arbitrary signal sets are given in $[6,11,20]$.

\section{Simulation results}

In this section we provide simulation results suggesting the behavior and performance of the OMF detector, in comparison to the MF detector. Although the simulation results do not prove that the OMF detector outperforms the MF detector, they suggest that the OMF detector may hold considerable promise in a variety of applications.

The behaviors of the detectors were simulated in non-Gaussian and Gaussian noise using random signal constellations. The signals in the constellation have dimension $m$ equal to the number of the signals in the constellation, and the samples of the signals are mutually independent zero-mean Gaussian random variables with variance $1 / \sqrt{m}$, scaled to have norm 1 .

We considered two different distributions for the non-Gaussian noise. The first is a Gaussian mixture of two components with equal weights. This choice of distribution is motivated by the fact that Gaussian mixtures have been used extensively to model non-Gaussian noise [16,24,28], and in part because the Gaussian mixture model is capable of closely approximating many non-Gaussian distributions. The second distribution is the Beta distribution, which is chosen since it is very flexible and capable of attaining a wide variety of shapes by varying its two parameter values $a$ and $b$. Depending on the values of these parameters the Beta distribution will have the "U", the "J", the triangle or the general bell shape. In addition, the
Beta distribution can model the effect of several noise components since the sum of $N$ Gamma-distributed random variables is Beta-distributed, if $N$ is not too large [3].

We generated 500 realizations of signals. For each particular signal realization, we determined the probability of correct detection for the detectors in both types of noise by recording the number of successful detections over 500 noise realizations. We then plotted histograms of the probability of correct detection $P_{d}$ for the different detectors, which indicated that $P_{d}$ has a unimodal distribution with a bell-shaped appearance. Therefore, it is reasonable to compactly present the results in terms of the mean and standard deviation of $P_{d}$ for the various detectors.

We note that the probability of correct detection levels we obtain in the simulations below are generally unacceptable in a communication context. However, we stress that the primary applications of the OMF detector are not in the context of communication, but rather other multi-signature scenarios such as target classification and signature analysis. In these contexts the probability of correct detection levels we obtain are useful. Applications of the ideas presented in this paper to a communication problem are considered in [8].

\subsection{Gaussian mixture noise}

We first considered a Gaussian mixture of two components each with standard deviation 0.25 centered at \pm 1 , corresponding to an SNR close to $0 \mathrm{~dB}$.

In Fig. 3 we plot the mean of $P_{d}$ for the OMF detector and the MF detector as a function of the number of signals in the transmitted constellation. The vertical lines indicate the standard deviation of $P_{d}$. From the figure it is evident that at this SNR the OMF detector outperforms the MF detector, where the relative improvement in performance of the OMF detector over the MF detector increases for increasing constellation size.

We repeated the simulations for different parameters of the Gaussian mixture components, again at an SNR of $0 \mathrm{~dB}$. In general we found that the relative improvement of the OMF detector over the MF detector increased as the separation between the mixtures increased. When the separation is decreased relative to the mixture standard deviation the relative 


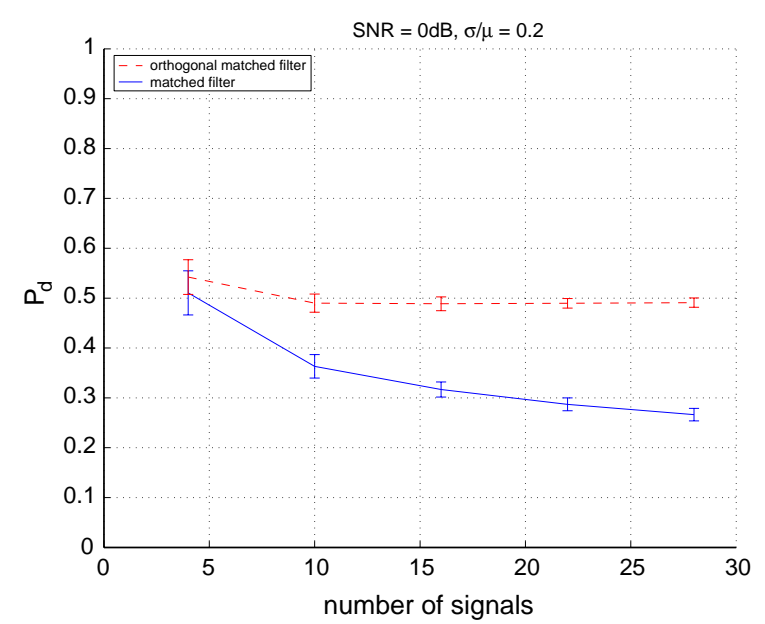

Fig. 3. Comparison between the OMF and MF in Gaussian mixture noise, as a function of the number of signals in the transmitted constellation. The mixture components have standard deviation of 0.25 and are centered at \pm 1 . The dashed line is the mean $P_{d}$ using the OMF detector, and the solid line is the mean $P_{d}$ using the MF detector. The vertical lines indicate the standard deviation of the corresponding $P_{d}$.

improvement in performance using the OMF detector decreases, consistent with the fact that the Gaussian mixture distribution approaches a Gaussian distribution. The same behavior is evident when varying the standard deviation of the mixture components for fixed mean separation. In Fig. 4 we plot the mean of $P_{d}$ for the OMF and MF detectors for constellations of 13 signals in Gaussian mixture noise of two components each with standard deviation $\sigma$ centered around $\pm \mu$, as a function of $\sigma / \mu$. The vertical lines indicate the standard deviation of $P_{d}$. As the standard deviation of the mixture components increases relative to the mixture mean, the Gaussian mixture distribution approaches a Gaussian distribution, in which case the relative improvement in performance using the OMF detector decreases.

In general we observed that the relative improvement in performance of the OMF over the MF detector increased with decreasing SNR, and is predominant for large signal constellation size. For increasing values of SNR the relative improvement in performance using the OMF detector decreases.

The qualitative behavior of the POMF detector in comparison to the MF detector when varying the

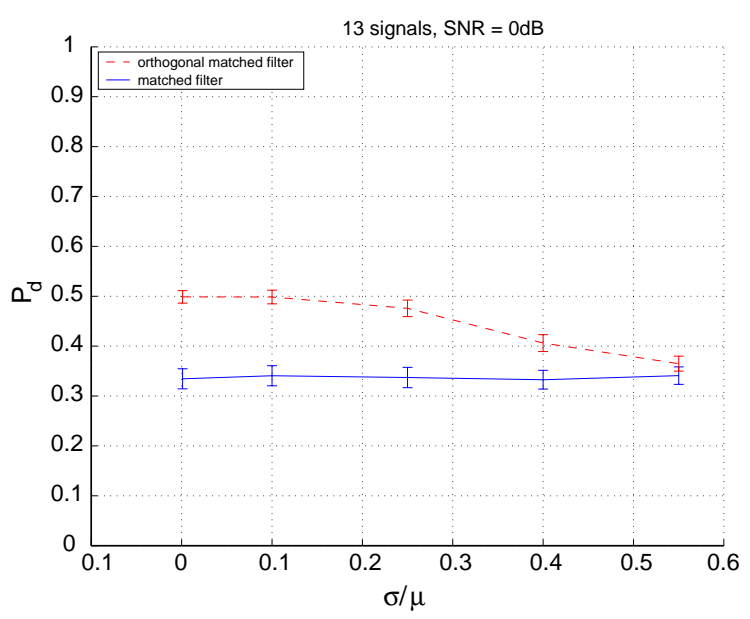

Fig. 4. Comparison between the OMF and MF detectors for transmitted constellations of 13 signals in Gaussian mixture noise with mixture components with standard deviation $\sigma$ centered at $\pm \mu$, as a function of $\sigma / \mu$. The dashed line is the mean $P_{d}$ using the OMF detector, and the solid line is the mean $P_{d}$ using the MF detector. The vertical lines indicate the standard deviation of the corresponding $P_{d}$.

Gaussian mixture parameters and the SNR is similar to that of the OMF detector.

\subsection{Beta distributed noise}

We next consider Beta-distributed noise with a variety of parameter values.

In Fig. 5 we plot the mean of $P_{d}$ for the OMF detector and the MF detector in Beta-distributed noise with $a=b=1$, as a function of the number of signals in the transmitted constellation. The vertical lines indicate the standard deviation of $P_{d}$. From the figure it is evident that the OMF detector outperforms the MF detector, where the relative improvement in performance of the OMF detector over the MF detector increases for increasing constellation size.

We repeated the simulations for different parameter values. In general we found that the relative improvement of the OMF detector over the MF detector increased as the distribution became more bimodal. In Figs. 6-8 we plot the mean of $P_{d}$ for the OMF and MF detectors for constellations of 13 signals in Beta-distributed noise with varying parameters. The vertical lines indicate the standard deviation of $P_{d}$. As 


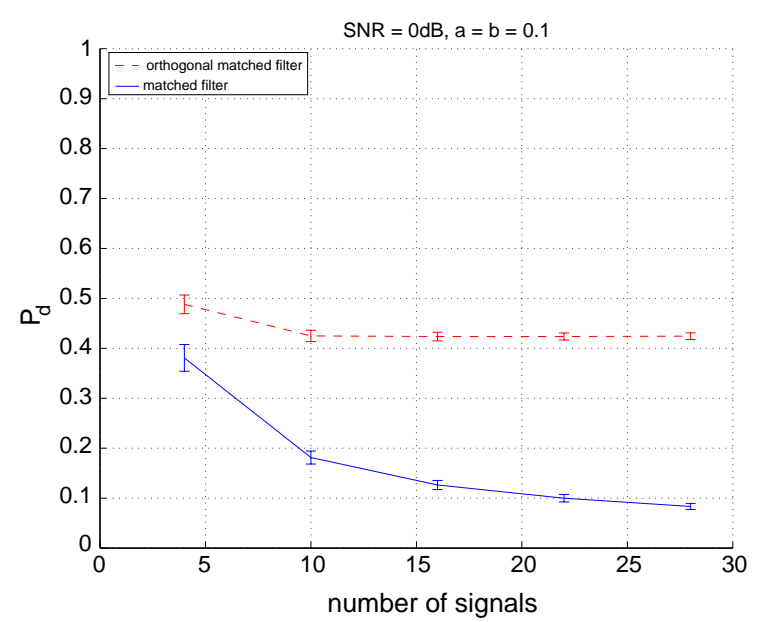

Fig. 5. Comparison between the OMF and MF detectors in Beta-distributed noise, as a function of the number of signals in the transmitted constellation. The parameters of the distribution are $a=b=0.1$. The dashed line is the mean $P_{d}$ using the OMF detector, and the solid line is the mean $P_{d}$ using the MF detector. The vertical lines indicate the standard deviation of the corresponding $P_{d}$.

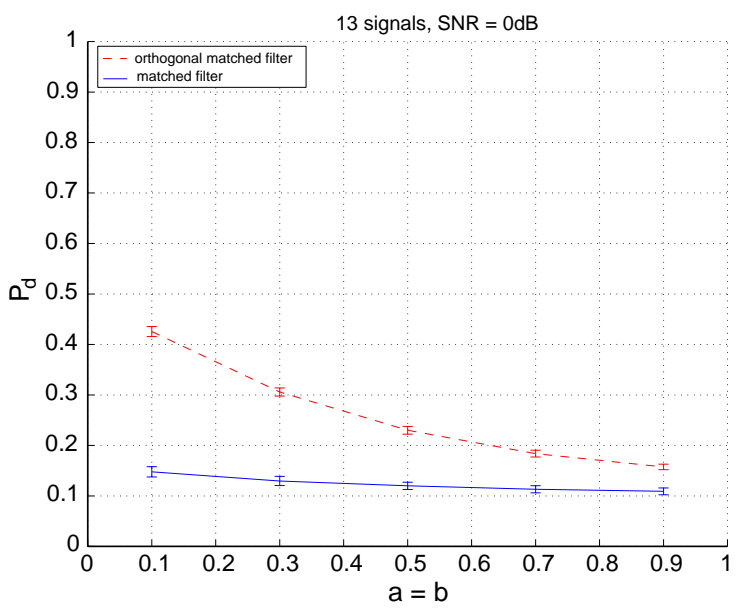

Fig. 6. Comparison between the OMF and MF detectors for transmitted constellations of 13 signals in Beta-distributed noise, as a function of the parameters with $a=b$. The dashed line is the mean $P_{d}$ using the OMF detector, and the solid line is the mean $P_{d}$ using the MF detector. The vertical lines indicate the standard deviation of the corresponding $P_{d}$.

the $b$ parameter increases, the Beta distribution approaches a unimodal distribution, in which case the relative improvement in performance using the OMF detector decreases.

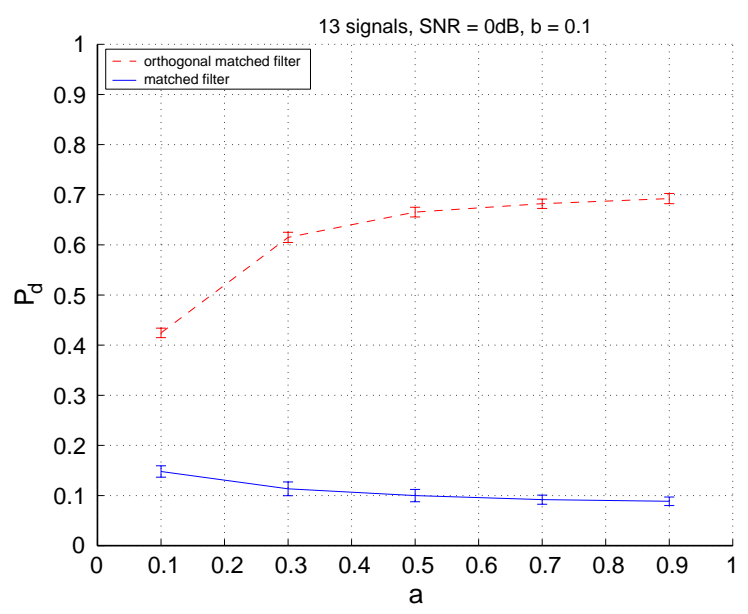

Fig. 7. Comparison between the OMF and MF detectors for transmitted constellations of 13 signals in Beta-distributed noise with $b=0.1$, as a function of the parameter $a$. The dashed line is the mean $P_{d}$ using the OMF detector, and the solid line is the mean $P_{d}$ using the MF detector. The vertical lines indicate the standard deviation of the corresponding $P_{d}$.

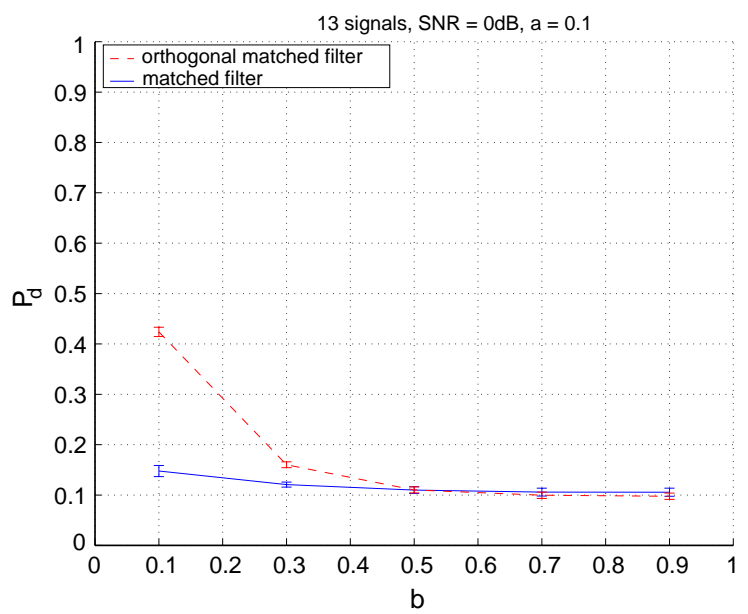

Fig. 8. Comparison between the OMF and MF detectors for transmitted constellations of 13 signals in Beta-distributed noise with $a=0.1$, as a function of the parameter $b$. The dashed line is the mean $P_{d}$ using the OMF detector, and the solid line is the mean $P_{d}$ using the MF detector. The vertical lines indicate the standard deviation of the corresponding $P_{d}$.

In Fig. 9 we plot the mean and standard deviation of $P_{d}$ for the OMF and MF detectors as a function of SNR for transmitted constellations of 13 signals, in Beta-distributed noise with $a=b=0.1$. The SNR is 


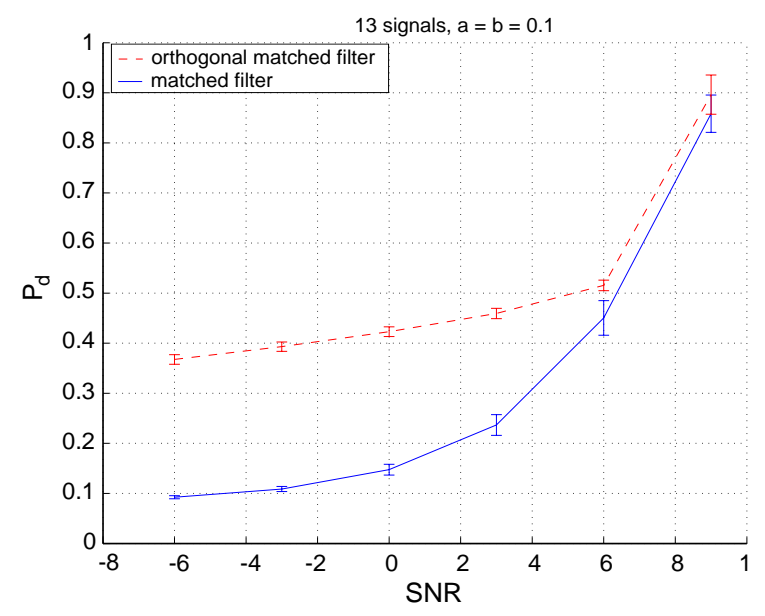

Fig. 9. Comparison between the OMF and MF detectors for transmitted constellations of 13 signals in Beta-distributed noise with $a=b=0.1$, as a function of the SNR. The dashed line is the mean $P_{d}$ using the OMF detector, and the solid line is the mean $P_{d}$ using the MF detector. The vertical lines indicate the standard deviation of the corresponding $P_{d}$.

given by $10 \log P_{S} / \sigma^{2}$, where $P_{S}$ is the signal power and the variance of the Beta distribution is given in terms of the parameters $a$ and $b$ as

$\sigma^{2}=\frac{a b}{(a+b)^{2}(a+b+1)}$.

The improvement in performance of the OMF over the MF detector is predominant for low to intermediate values of SNR.

\subsection{Gaussian noise}

We repeated the simulations leading to the results previously presented with zero-mean Gaussian noise. In Fig. 10 we plot the mean of $P_{d}$ for the OMF detector and the MF detector in Gaussian noise, as a function of the number of signals in the transmitted constellation. The vertical lines indicate the standard deviation of $P_{d}$. In Fig. 11 we plot the mean of $P_{d}$ using the OMF and MF detectors for transmitted constellations of 7 signals in Gaussian noise, as a function of SNR.

As expected, for Gaussian noise the MF detector outperforms the OMF detector. This is consistent with the fact that the MF detector maximizes the probability of correct detection for Gaussian noise. However, it is evident from Figs. 10 and 11 that the relative

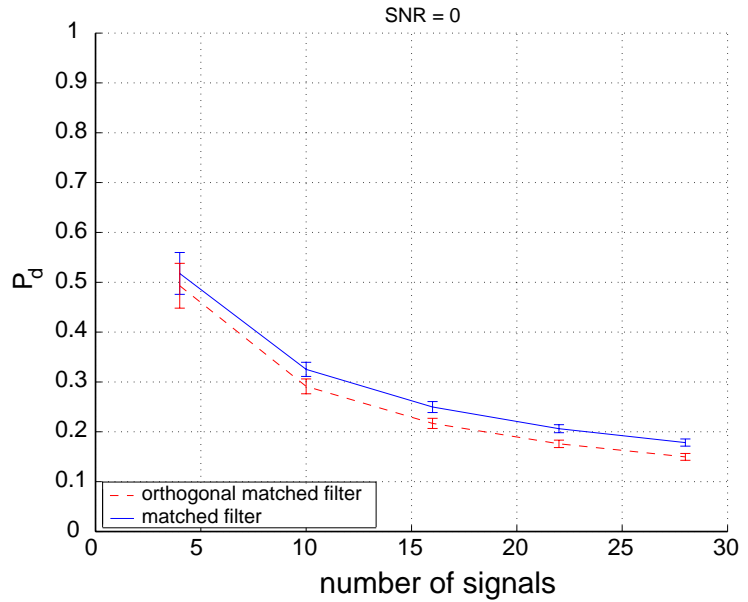

Fig. 10. Comparison between the OMF and MF detectors in zero mean, unit variance Gaussian noise, as a function of the number of signals in the transmitted constellation. The dashed line is the mean $P_{d}$ using the OMF detector, and the solid line is the mean $P_{d}$ using the MF detector. The vertical lines indicate the standard deviation of the corresponding $P_{d}$.

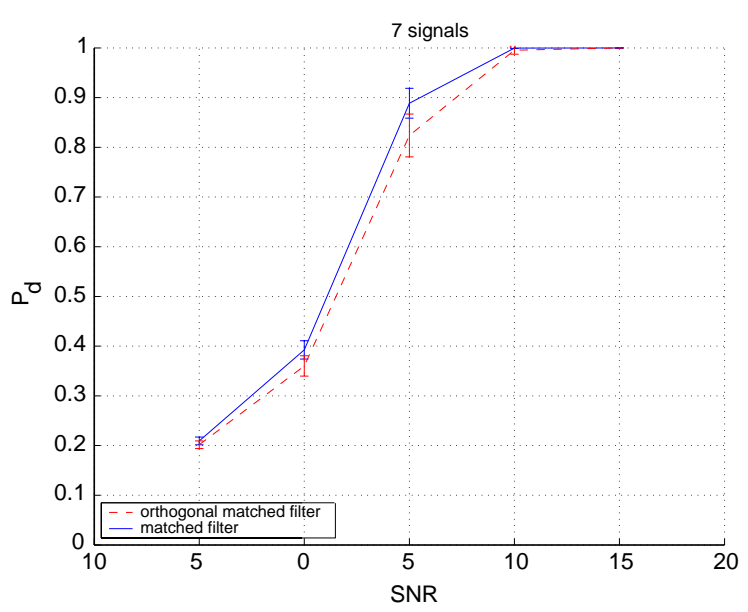

Fig. 11. Comparison between the OMF and MF detectors for transmitted constellations of 7 signals in Gaussian noise, as a function of SNR. The dashed line is the mean $P_{d}$ using the OMF detector, and the solid line is the mean $P_{d}$ using the MF detector. The vertical lines indicate the standard deviation of the corresponding $P_{d}$.

improvement in performance using the MF detector over the OMF is not very significant. Specifically, in Fig. 11 note that the maximum (mean) difference in probability of correct detection is less than 0.08 . These 
results are encouraging since they suggest that if the receiver is designed to operate in different noise environments, or in an unknown noise environment, than we may prefer using an OMF or POMF detector since for certain non-Gaussian noise distributions these detectors may result in a substantial improvement in performance over a MF detector, without significantly degrading the performance if the noise is Gaussian.

The performance trend of the OMF relative to that of the MF for the Gaussian case generally holds for other distributions that are unimodal, even when the distribution is asymmetric or has heavy tails.

\section{Conclusion}

We considered the problem of detecting a transmitted signal when one of $m$ known signals is transmitted over a white noise channel. To improve the performance over MF detection for non-Gaussian noise, we proposed whitening the output of the MF demodulator on an appropriate subspace, prior to detection. The whitening transformation was chosen to minimize the MSE between the input and the output.

If the transmitted signals are linearly independent, then this approach results in a demodulator that is equivalent to a correlation demodulator with orthonormal signals that are closest in a least-squares sense to the transmitted signals, and is therefore referred to as an OMF demodulator. If the transmitted signals are linearly dependent, then this approach results in a demodulator that is equivalent to a correlation demodulator with signals that form a projected orthogonal basis for the space spanned by the transmitted signals, and is closest in a least-squares sense to the transmitted signals. This demodulator is therefore referred to as a POMF demodulator.

We provided simulations that suggest that the OMF and POMF detectors significantly outperform the MF detector for some classes of non-Gaussian noise. When the noise is Gaussian the loss in performance using the OMF and POMF detectors in comparison with the MF detector is negligible.

In this paper, the performance improvement using the OMF receiver was demonstrated through simulation only. An important direction for future research is to analyze the behavior of the OMF receiver analytically. Of related interest is the development of analytical methods for determining under which non-Gaussian distributions the modified receivers lead to improved performance over the MF receiver, for example using large deviation theory and the Chernoff bound.

In our closing remarks, we note that we can readily extend the results developed in this paper to the case in which the signals $s_{k}(t)$ have unequal norm, or the case in which the signals are transmitted with unequal probability. In such cases, we may choose the correlating signals $q_{k}(t)$ to minimize a weighted least-squares error, where the weights may be chosen to reflect the signal priors, or the signal norms.

\section{Appendix A. Orthonormal set transformations}

In this appendix we prove that a set transformation $X$ corresponding to the signals $\left\{x_{k}(t), 1 \leqslant k \leqslant m\right\}$ satisfies $X^{*} X=\mathbf{I}_{m}$ if and only if the signals $x_{k}(t)$ are orthonormal.

Suppose the signals $x_{k}(t)$ are orthonormal. For any $\mathbf{a} \in \mathscr{R}^{m}$, let $y(t)=X \mathbf{a}$ and $\mathbf{b}=X^{*} y(t)=X^{*} X \mathbf{a}$. Then

$$
\begin{aligned}
b_{l} & =\left\langle x_{l}(t), y(t)\right\rangle=\left\langle x_{l}(t), \sum_{k=1}^{m} a_{k} x_{k}(t)\right\rangle \\
& =\sum_{k=1}^{m} a_{k}\left\langle x_{l}(t), x_{k}(t)\right\rangle \\
& =\sum_{k=1}^{m} a_{k} \delta_{l k}=a_{l} .
\end{aligned}
$$

Since $b_{l}=a_{l}$ for all $l, X^{*} X \mathbf{a}=\mathbf{a}$ for any $\mathbf{a} \in \mathscr{R}^{m}$; thus $X^{*} X=\mathbf{I}_{m}$.

Next, suppose $X^{*} X=\mathbf{I}_{m}$. Then $X^{*} X \mathbf{a}=\mathbf{a}$ for any $\mathbf{a} \in \mathscr{R}^{m}$. In particular, let $\mathbf{a}=\mathbf{e}_{l}$, where $\mathbf{e}_{l}$ is the vector with $k$ th component $\delta_{k l}$. Then

$X^{*} X \mathbf{e}_{l}=\mathbf{e}_{l}, \quad 1 \leqslant l \leqslant m$.

Now,

$X \mathbf{e}_{l}=\sum_{k=1}^{m} x_{k}(t) \delta_{k l}=x_{l}(t)$.

Substituting (A.3) into (A.2) we have

$X^{*} x_{l}(t)=\mathbf{e}_{l}, \quad 1 \leqslant l \leqslant m$. 
Since the $k$ th element of $X^{*} x_{l}(t)$ is given by $\left\langle x_{k}(t), x_{l}(t)\right\rangle$, from (A.4) we conclude that

$\left\langle x_{k}(t), x_{l}(t)\right\rangle=\delta_{k l}, \quad 1 \leqslant k, l \leqslant m$,

and the signals $x_{k}(t)$ are orthonormal.

\section{Appendix B. Equivalence of minimizing $\epsilon_{\mathrm{LS}}\left(\left\{s_{k}(t)\right\}\right.$, $\left.\left\{f_{k}(t)\right\}\right)$ and maximizing $R\left(\left\{s_{k}(t)\right\},\left\{f_{k}(t)\right\}\right)$}

Expanding $\varepsilon_{\mathrm{LS}}\left(\left\{s_{k}(t)\right\},\left\{f_{k}(t)\right\}\right)$ we have

$$
\begin{aligned}
\varepsilon_{\mathrm{LS}} & \left(\left\{s_{k}(t)\right\},\left\{f_{k}(t)\right\}\right) \\
= & \sum_{k=1}^{m}\left\langle s_{k}(t)-f_{k}(t), s_{k}(t)-f_{k}(t)\right\rangle \\
= & \sum_{k=1}^{m}\left(1+\left\langle f_{k}(t), f_{k}(t)\right\rangle\right. \\
& \left.-2\left\langle s_{k}(t), f_{k}(t)\right\rangle\right) .
\end{aligned}
$$

Since the signals $\left\{f_{k}(t), 1 \leqslant k \leqslant m\right\}$ form a POB for the $n$-dimensional space $\mathscr{U} \subseteq \mathscr{H}, f_{k}(t)=P_{\mathscr{U}} x_{k}(t)$ for some orthonormal set of signals $\left\{x_{k}(t), 1 \leqslant k \leqslant m\right\}$. Let $F: \mathscr{R}^{m} \rightarrow \mathscr{H}$ and $X: \mathscr{R}^{m} \rightarrow \mathscr{H}$ denote the set transformations corresponding to $f_{k}(t)$ and $x_{k}(t)$, respectively. Then, $F=P_{\mathscr{U}} X$ and

$\sum_{k=1}^{m}\left\langle f_{k}(t), f_{k}(t)\right\rangle=\operatorname{Tr}\left(F^{*} F\right)=\operatorname{Tr}\left(X^{*} P_{\mathscr{U}} X\right)$.

Let the signals $\left\{z_{k}(t), 1 \leqslant k \leqslant n\right\}$ be a set of orthonormal signals that span $\mathscr{U}$, and let $Z$ be the set transformation corresponding to the signals $z_{k}(t)$. Then $P_{\mathscr{U}}=Z Z^{*}$. Since $\mathscr{U}$ is a subset of the span of the vectors $x_{k}(t)$, any $z_{k}(t)$ can be expressed as $z_{k}(t)=X \mathbf{Z}_{k}$ for some $\mathbf{Z}_{k} \in \mathscr{R}^{m}$. Thus, $Z=X \mathbf{Z}$ where $\mathbf{Z}$ is the matrix of columns $\mathbf{Z}_{k}$. Since the vectors $x_{k}(t)$ and $z_{k}(t)$ are orthonormal, $X^{*} X=I_{m}$ and $Z^{*} Z=I_{n}$. But $Z^{*} Z=\mathbf{Z}^{*} X^{*} X \mathbf{Z}=\mathbf{Z}^{*} \mathbf{Z}$; so $\mathbf{Z}^{*} \mathbf{Z}=I_{n}$. Then

$$
\begin{aligned}
\sum_{k=1}^{m}\left\langle f_{k}(t), f_{k}(t)\right\rangle & =\operatorname{Tr}\left(X^{*} P_{\mathscr{U}} X\right) \\
& =\operatorname{Tr}\left(X^{*} X \mathbf{Z} \mathbf{Z}^{*} X^{*} X\right) \\
& =\operatorname{Tr}\left(I_{n}\right)=n,
\end{aligned}
$$

so that minimizing $\varepsilon_{\mathrm{LS}}\left(\left\{s_{k}(t)\right\},\left\{f_{k}(t)\right\}\right)$ is equivalent to maximizing $R\left(\left\{s_{k}(t)\right\},\left\{f_{k}(t)\right\}\right)$.

\section{References}

[1] A. Aldroubi, Portraits of frames, Proc. Amer. Math. Soc. 123 (1995) 1661-1668.

[2] M. Artin, Algebra, Prentice-Hall, Upper Saddle River, NJ, 1991.

[3] D. D'Addio, S. Giannatempo, G. Galati, Generation of K-distributed random variables, Trans. Society Comp. Simulation 5 (1988) 159-174.

[4] I. Daubechies, Ten Lectures on Wavelets, SIAM, Philadelphia, PA, 1992.

[5] Y.C. Eldar, Quantum signal processing, Ph.D. Thesis, Massachusetts Institute of Technology, 2001.

[6] Y.C. Eldar, Least-squares inner product shaping, Linear Alg. Appl. 348 (May 2002) 153-174.

[7] Y.C. Eldar, H. Bölcskei, Geometrically uniform frames, IEEE Trans. Inform. Theory 49 (April 2003) 993-1006.

[8] Y.C. Eldar, A.M. Chan, An optimal whitening approach to linear multiuser detection, IEEE Trans. Inform. Theory 49 (September 2003) 2156-2171.

[9] Y.C. Eldar, G.D. Forney Jr., On quantum detection and the square-root measurement, IEEE Trans. Inform. Theory 47 (March 2001) 858-872.

[10] Y.C. Eldar, G.D. Forney Jr., Optimal tight frames and quantum measurement, IEEE Trans. Inform. Theory 48 (March 2002) 599-610.

[11] Y.C. Eldar, A. Megretski, G.C. Verghese, Designing optimal quantum detectors via semidefinite programming, IEEE Trans. Inform. Theory 49 (April 2003) 1012-1017.

[12] Y.C. Eldar, A. Megretski, G.C. Verghese, Optimal detection of symmetric mixed quantum states, IEEE Trans. Inform. Theory, in press.

[13] Y.C. Eldar, A.V. Oppenheim, Orthogonal matched filter detection, in: Proceedings of the International Conference on Acoustics, Speech, Signal Processing (ICASSP-2001), Salt Lake, UT, May 2001.

[14] Y.C. Eldar, A.V. Oppenheim, Orthogonal multiuser detection, Signal Processing 82 (2002) 321-325.

[15] Y.C. Eldar, A.V. Oppenheim, MMSE whitening and subspace whitening, IEEE Trans. Inform. Theory 49 (July 2003) 1846-1851.

[16] B.S. Everitt, D.J. Hand, Finite Mixture Distributions, Chapman \& Hall, London, UK, 1981.

[17] G.D. Forney Jr., Geometrically uniform codes, IEEE Trans. Inform. Theory 37 (5) (September 1991) 1241-1260.

[18] E.M. Friel, K.M. Pasala, Direction finding with compensation for a near field scatterer, in: International Symposium Antennas and Propagation Society, 1995, pp. 106-109.

[19] G.H. Golub, C.F. Van Loan, Matrix Computations, 3rd Edition, Johns Hopkins University Press, Baltimore MD, 1996.

[20] C.W. Helstrom, Bayes-cost reduction algorithm in quantum hypothesis testing, IEEE Trans. Inform. Theory 28 (March 1982) 359-366.

[21] N.J. Higham, Computing the polar decomposition-with applications, SIAM J. Sci. Stat. Comput. 7 (1986) $1160-1174$ 
[22] R.A. Horn, C.R. Johnson, Matrix Analysis, Cambridge University Press, Cambridge, UK, 1985.

[23] T. Kailath, H.V. Poor, Detection of stochastic processes, IEEE Trans. Inform. Theory 44 (October 1998) 2230-2231.

[24] G.J. McLachlan, K.E. Basford, Mixture Models, Marcel Dekker, New York, 1988.

[25] R.J. Piechocki, N. Canagarajah, J.P. McGeehan, Improving the direction-of-arrival resolution via double code filtering in WCDMA, in: First International Conference on 3G Mobile Communication Technologies, March 2000, pp. 204-207.

[26] J.G. Proakis, Digital Communications, 3rd Edition, McGraw-Hill, New York, 1995.
[27] L.L. Scharf, Statistical Signal Processing: Detection, Estimation and Time Series Analysis, Addison-Wesley, Reading, MA, 1991.

[28] D.M. Titterington, A.F.M. Smith, U.E. Makov, Statistical Analysis of Finite Mixture Distributions, Wiley, Chichester, UK, 1985.

[29] P. Zielinski, K. Zietak, The polar decomposition - properties, applications and algorithms, Matematyka Stosowana 38 (1995) 23-40. 